九州大学学術情報リポジトリ

Kyushu University Institutional Repository

\title{
Distribution of Asphondylia Gall Midges (Diptera: Cecidomyi idae) in Japan
}

Uechi, Nami

Tokuda, Makoto

Yukawa, Jun-ichi

https://doi.org/10.5109/2656

出版情報: ESAKIA. 42, pp.1-10，2002-03-31. Entomological Laboratory, Faculty of Agriculture, Kyushu University

バージョン :

権利関係 : 


\title{
Distribution of Asphondylia Gall Midges (Diptera: Cecidomyiidae) in Japan*
}

\author{
Nami UECHI, Makoto TOKUDA \\ Entomological Laboratory, Graduate School of Bioresource and Bioenvironmental Sciences, \\ Kyushu University, Fukuoka, 812-8581 Japan \\ and \\ Junichi YUKAWA \\ Entomological Laboratory, Faculty of Agriculture, \\ Kyushu University, Fukuoka, 812-8581 Japan
}

\begin{abstract}
Either summer or winter hosts have been known for the Japanese multivoltine gall midges of the genus Asphondylia. We renew the distribution records of these gall midges based on more recent and detailed collecting data that have been accumulated since Yukawa \& Masuda (1996). Distributional information of each Asphondylia gall midge is quite important to combine gall midges utilizing summer hosts with those utilizing winter hosts, because host alternation by the gall midges has been suspected. The distributional information indicated two possible combinations between spring-summer and autumn-winter gall midges.
\end{abstract}

Key words: Asphondylia, Cecidomyiidae, gall, distributional information, multivoltine, host plant.

\section{Introduction}

In Japan, Asphondylia gall midges have been collected from at least 20 plant species of 18 families (Yukawa, 1971; Yukawa, 1982; Yukawa \& Masuda, 1996; Table 1). Most of these species are multivoltine and cannot complete their annual life history on a single

* Contribution from the Entomological Laboratory, Faculty of Agriculture, Kyushu University, Fukuoka (Ser. 5, No.79). 
host plant, because plant organs for oviposition do not exist on their respective host plants at the adult emergence season. Therefore, they have to find both summer and winter hosts that are utilized alternately in a year. Our recent field survey and DNA analysis suggest the possibility of host alternation by these gall midges (Yukawa et al., in preparation). In addition to these survey and analysis, the distributional information of each gall midge is quite important to combine summer and winter hosts that are necessary to coexist in the distribution range of respective gall midges.

The distributional information of the Japanese Asphondylia gall midges was roughly indicated in Yukawa \& Masuda (1996). In the present paper, we renew the distribution records of the Japanese Asphondylia gall midges based on more recent and detailed collecting data that our colleagues and we have been accumulating since 1996.

\section{Materials and Methods}

We collected fruit or bud galls produced by Asphondylia gall midges on their respective host plants at various localities in Japan. For identification, some of the collected galls were dissected under a binocular microscope to obtain larval and pupal specimens. Remaining galls were maintained in plastic containers $(10 \mathrm{~cm}$ in diameter, $6 \mathrm{~cm}$ in depth) to rear adult midges. Mature larvae, pupae or emerged adults were put into $70-75 \%$ ethanol for morphological observation or $99.5 \%$ acetone for DNA analysis. In addition to our own collecting data, we referred to distributional information from our colleagues.

In the present list, we arranged the collecting records of Asphondylia midge galls according to the usual systematic order of plant families. Respective collecting records consist of galled part, host plant, locality, collecting date, collector, and some other biological information. The names of Japanese main islands are abbreviated in brackets as HK (Hokkaido), HS (Honshu), SH (Shikoku), KY (Kyushu), and SW (the Southwestern Islands south of Yakushima Is.), and followed by the names of prefectures. The names of locality with an asterisk mean that the galls have previously been collected from that place, otherwise they were collected there for the first time. The collecting dates with a plus mark mean that the galls were collected there more than one time.

Some of collector's names are abbreviated as follows: JY (J. Yukawa), NU (N. Uechi), MT (M. Tokuda), and SU (S. Usuba).

\section{Results}

\section{MORACEAE}

Axillary bud gall on Morus alba L. by Asphondylia morivollera (Naito)

[HK]: Hokkaido Univ., Sapporo City*, 26 Jun. 2001, JY; Tonebetsu, Iwamizawa City, 17 Jun. 2001, S. Akimoto. 
Most of galls collected in 2001 were parasitized by eurytomid wasps.

\section{HAMAMELIDACEAE}

Fruit gall on Distylium racemosum Sieb. et Zucc. (Fig.1A) by Asphondylia sp.

[KY: Fukuoka]: Kyushu Univ. Forest, Sasaguri Town, 19 Aug. 2001+, NU; [SW:

Okinawa]: Morinokawa Park, Ginowan City, 4 Mar. 2001, NU; Yona, Kunigami Vil., 24 Mar. 2000*, NU; Hijigawa River, Kunigami Vil., 24 Feb. 2001, JY; Mt. Bannadake, Ishigaki City, 26 Feb. 2001, NU.

This gall was recorded previously only on Okinawa Is. We added the collection records from Ishigaki Is. and Fukuoka, for the first time.

\section{ANACARDIACEAE}

Flower bud gall on Rhus succedanea L. by Asphondylia sp.

[KY: Kumamoto]: Suya, Nishigoshi Town, 7 Jun. 2000, M. Okamura.

Flower bud gall on Rhus sylvestris Sieb. et Zucc by Asphondylia sp.

[SH: Tokushima]: Kamiyama Forest Park, Kamiyama Town, Jun. 2001, M. Yukinari;

[KY: Fukuoka]: Aobanomori Park, Fukuoka City, 10 Jun. 2001+, NU; Minami Park, Fukuoka City, 29 Jun. 2000+, NU.

\section{CELASTRACEAE}

Fruit gall on Celastrus orbiculatus Thunb. by Asphondylia sp.

[KY: Fukuoka]: Mt. Kusenbusan, Nakagawa Town, 12 Aug. 2001+, NU.

\section{VITACEAE}

Fruit gall on Ampelopsis brevipedunculata (Maxim.) Trautv. by Asphondylia baca Monzen

[HK]: Hokkaido Univ. Botanical Garden, Sapporo City, 4 Sep. 1999*, NU; Ohiso, Abuta Town, 6 Sep. 1999, NU; Ohnuma, Nanae Town, 5 Sep. 1999, NU; [HS: Miyagi]: Nuruyu, Hanayama Vil., 17. Sep. 2001, JY; Izunuma, Wakayanagi Town, 18 Sep. 2001, JY; [SH: Tokushima]: Kamiyama Forest Park, Kamiyama Town, 22 Sep. 2001, M. Yukinari; [SH: Kochi]: Murotomisaki, Muroto City, 28 Sep. 1999, NU; [KY: Fukuoka]: Aobanomori Park, Fukuoka City, 29 Jun. 2001+, NU; Mt. Konomiyama, Fukuma Town, 19 Sep. 1999, JY; Ino, Hisayama Town, 6 Nov. 1998+, NU; Inunaki, Wakamiya Town, 8 Jul. 1999+, NU; Hoshino Vil., Yame, 22 Aug. 1999, NU; Mt. Kusenbusan, Nakagawa Town, 7 Sep. 2001, NU; Mt. Hikosan, Soeda Town, 17 Jul. 1998, NU; Sharikura, Fukuma Town, 19 Sep. 1999, NU; Shikanoshima Is., Fukuoka City, 7 Jul. 1999+, NU; Fukuoka City, 14 Jul. 1999, NU; [KY: Oita]: Yufuin Town, 4 Aug. 1999, M. Shoubu; Inoseto, Beppu City, 31 Aug. 2001, JY.

Fruit gall on Ampelopsis brevipedunculata forma glabrifolia (Honda) Kitamura by Asphondylia baca Monzen

[SW: Okinawa]: Banna Park, Ishigaki City, 2 Mar. 2001+, NU.

This is the first record of the fruit gall produced by Asphondylia baca on Ishigaki Is. Also see remarks. 
Fruit gall on Cayratia japonica (Thunb.) Gagnep by Asphondylia sp.

[HS: Miyagi]: Tohoku Univ., Sendai City, 21 Sep. 2001, NU; [KY: Fukuoka]: Kyushu Univ. Forest, Sasaguri Town, 19 Sep. 2000, NU.

\section{CORNACEAE}

Fruit gall on Aucuba japonica Thunb. by Asphondylia aucubae Yukawa et Ohsaki

[HS: Miyagi]: Aoba Castle, Sendai City; 20 Sep. 2001, NU; [HS: Tokyo]: FukiageGyoen, the Imperial Palace, 28 May 1998+, JY; [HS: Shiga]: Hikone Castle, Hikone City, 19 Sep. 1998, JY; [HS: Kyoto]: Kwasan Observatory, Kyoto City, 12 Mar. 2000, NU; Kyoto Univ. Botanical Garden, Kyoto City, 12 Mar. 2000, NU; Higashiyama, Kyoto City, 14 Mar. 2000, NU; Mt. Yoshidayama, Kyoto City, 12 Mar. 2000, NU; [HS: Wakayama]: Takinokoto, Susami Town, 6 May 2001, M. Shoubu; [HS: Shimane]: Hitokubota, Sada Town, 2 Apr. 2001. MT; Kamiko, Hamada City, 2 Apr. 2001. MT; [HS: Yamaguchi]: Hagi City, 3 Apr. 2001, JY; Omine-cho, Mine City, 3 Jan. 2002, MT; [SH: Kochi]: Osugi, Otoyo Town, 5 Dec. 2001, MT; Kamiyoshihara, Kagami Vil., 5 Dec. 2001, MT; [KY: Fukuoka]: Mt. Tachibanayama, Fukuoka City, 22 Feb. 2001, NU; Ino, Hisayama Town, 9 Mar. 1998+, NU; Mt. Hikosan, Soeda Town, May 1999+, NU; Mt. Konomiyama, Fukuma Town, 21 Aug. 1999, JY; [KY: Saga]: Matsukuma, Higashisefuri Vil., 9 Jun. 2001, NU; [KY: Nagasaki]: Kiba, Chijiwa Town, 22 Apr. 2000, NU; [KY: Oita]: Usa-hachimangu, Usa City, 19 Sep. 1997, JY; [KY: Kumamoto]: Otsuka, Hitoyoshi City, 4 May 2001, JY; [KY: Miyazaki]: Aya Town, 1 Dec. 2001, JY; Miyazaki-jingu, Miyazaki City, 10 Mar. 2000, D. Yamaguchi; [KY: Kagoshima]: Shiroyama, Kagoshima City*, 10 Apr. 2000, JY; Ono, Kagoshima City, 7 Mar. 1999, JY; Mt. Takakumayama, Kanoya City, 12 Apr. 2000, JY.

Fruit gall on Aucuba japonica var. borealis Miyabe et Kudo by Asphondylia aucubae Yukawa et Ohsaki

[HS: Aomori]: Mt. Bonjusan, Aomori City, 7 Jun. 2000, E. Kimura; Moya, Aomori City, 19 Jun. 2000, E. Kimura; [HS: Niigata]: Mt. Gozusan, Sasakami Vil., 18 May 2000, S. Sato.

Fruit gall on Helwingia japonica (Thunb.) F. G. Dietr. by Asphondylia sp.

[HS: Yamanashi]: Hirogawara, Ashiyasu Vil., 12 Jul. 2001, H. Inoue; [HS: Kyoto]: Iwakura-Muramatsu, Kyoto City, 5 Jun. 1999, H. Inoue; [KY: Fukuoka]: Mt. Hikosan, Soeda Town, 21 Jun. 2000, NU; [KY: Kagoshima]: Terayama, Kagoshima City, 12 Apr. 2000*, JY.

This gall was recorded previously only in Kagoshima Prefecture. We added the collection records from Yamanashi, Kyoto and Fukuoka Prefectures, for the first time. 


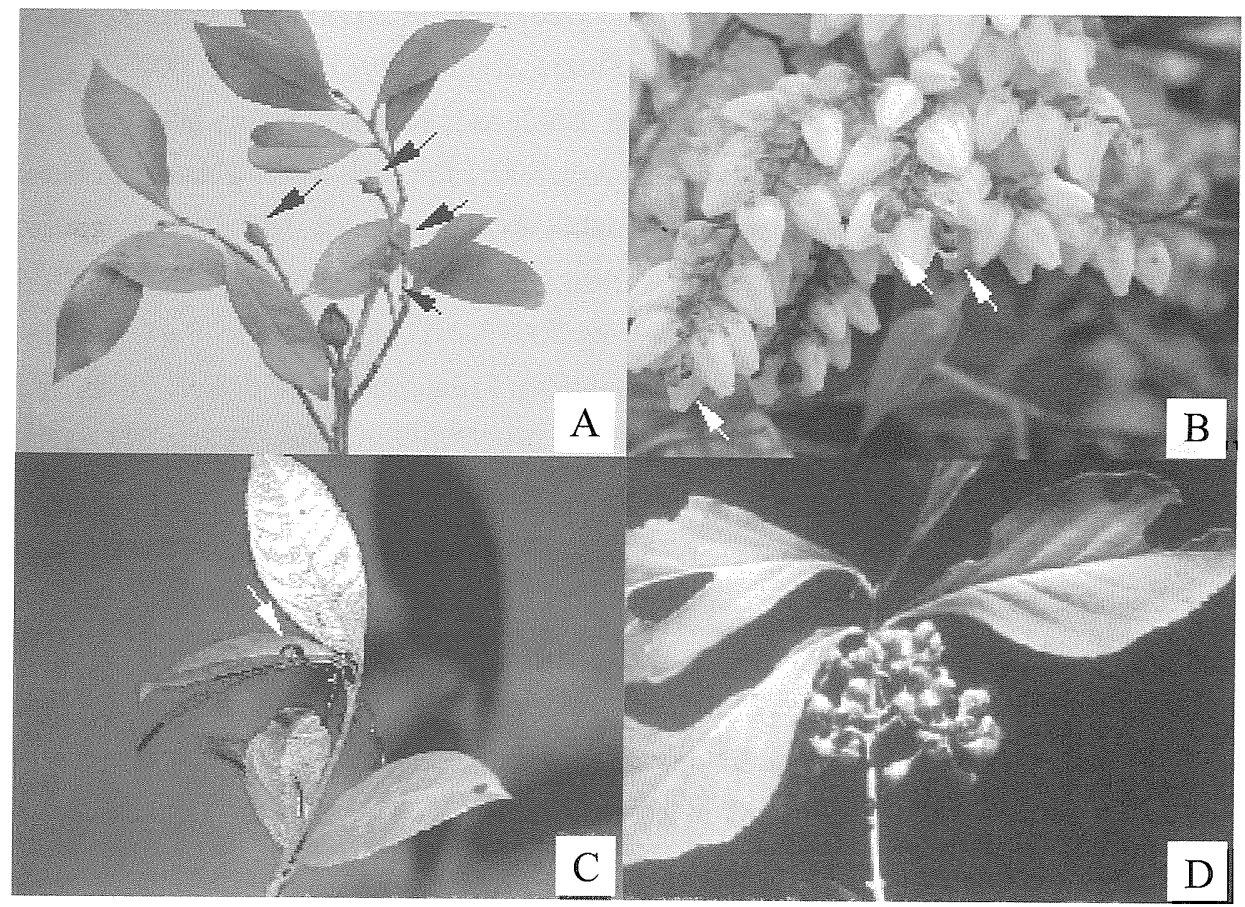

Figs. 1A-D: Galls produced by Asphondylia gall midges; A: fruit galls on Distylium racemosum [HAMAMELIDACEAE]; B: flower bud galls on Pieris japonica [ERICACEAE]; C: Fruit gall on Ardisia japonica [MYRSINACEAE]; D: Fruit galls on Callicarpa japonica [VERBENACEAE] (The fruit was all transformed into galls).

Flower bud gall on Pieris japonica D. Don (Fig. 1B) by Asphondylia sp.

[HS: Miyagi]: Aoba Castle, Sendai City, 20 Sep. 2001, NU; [HS: Tokyo]: Tokyo Univ. of Agr., Tokyo, 30 Mar. 2002, NU; [HS: Kyoto]: Kwasan Observatory, Kyoto City, 12 Mar. 2000, NU; [KY: Fukuoka]: Minami Park, Fukuoka City, 5 Mar. 2000+, NU; [KY: Kagoshima]: Mt. Takakumayama, Kanoya City,12 Apr. 2000, JY. MYRSINACEAE

Fruit gall on Ardisia japonica (Thunb.) B1. (Fig. 1C) by Asphondylia sp.

[HS: Miyagi]: Yasoen, Mogasaki, Sendai City, 22 Sep. 2001, NU; [HS: Niigata]: Joetsu Univ. of Education, Joetsu City, 12 May 2000+, Y. Iokawa; [HS: Saitama]: Komahongo, Hidaka City, 2 Nov. 1980*, SU; Nenogongen, Hanno City, 25 May 1981, SU; [KY: Fukuoka]: Ino, Hisayama Town, 31 May 2001+, NU; Oronoshima Is., 26 May 2001, NU; Kyushu Univ. Forest, Sasaguri Town, 19 Aug. 2001+, NU. 
Fruit gall on Ligustrum japonicum Thunb. by Asphondylia sphaera Monzen [HS: Shiga]: Hikone Castle, Hikone City, 3 Oct. 1998, JY; [HS: Kyoto]: Kyoto Univ., Kyoto City, 13 Mar. 2000, NU; Yoshida Shrine, Kyoto City, 13 Mar. 2000, NU; [HS: Shimane]: Hitokubota, Sada Town, 2 Apr. 2002, MT; Kamiko, Hamada City, 2 Apr. 2002, MT; [HS: Yamaguchi]: Hagi City, 3 Apr. 2002, JY; [SH: Kochi]: Anauchi, Otoyo Town, 5 Dec. 2001, MT; Katsurahama, Kochi City, 5 Dec. 2001, MT; [KY: Fukuoka]: Yakuoji, Koga City, 30 Apr. 1999, NU; Oronoshima Is., Fukuoka City, 26 May 2001, NU; Shikanoshima Is., Fukuoka City, 4 May 2001+, NU; Aobanomori Park, Fukuoka City, 29 Jun. 2000+, NU; Mt. Tachibanayama, Fukuoka City, 26 Apr. 2000, NU; Nagatani Park, Fukuoka City, 6 Mar. 1999+, NU; Maizuru Park, Fukuoka City, 15 Mar. 2001+, NU; Minami Park, Fukuoka City, 18 Jun. 2001+, NU; Ino, Hisayama Town, 9 Mar. 1998+, NU; Inunaki, Wakamiya Town, 8 Jul. 1999+, NU; Kyushu Univ. Forest, Sasaguri Town, 10 Apr. 2001+, NU; [KY: Nagasaki]: Hirado City, 23 Mar. 1983, SU; [KY: Saga]: Matsukuma, Higashisefuri Vil., 9 Jun. 2001, NU; [KY: Kagoshima]: Terayama, Kagoshima City, 10 Apr. 2000*, JY; Shiroyama, Kagoshima City, 7 Mar. 1999*, NU; Shimode, Kagoshima City, 9 Jun. 2000*, JY; Fukiagehama, Fukiage Town, 22 Jul. 2001, NU; [SW: Okinawa]: Urasoe-Joushi, Urasoe City, 3 Mar. 2001, NU.

Flower bud gall on Ligustrum japonicum Thunb. by Asphondylia sp.

[KY: Fukuoka]: Aobanomori Park, Fukuoka City, 29 Jun. 2000+, NU; Mt. Tachibanayama, Fukuoka City, 26 Apr. 2000, NU; Nagatani Park, Fukuoka City, 6 Mar. 1999+, NU; Ino, Hisayama Town, 9 Mar. 1998+, NU; Kyushu Univ. Forest, Sasaguri Town, 10 Apr. 2001+, NU; [KY: Saga]: Matsukuma, Higashisefuri Vil., 9 Jun. 2001, NU; [KY: Kagoshima]: Terayama, Kagoshima City, 10 Apr. 2000*, JY; Shiroyama, Kagoshima City, 7 Mar. 1999*, NU; Shimode, Kagoshima City, 9 Jun. $2000^{*}, J Y$.

An unidentified gall midge producing flower bud galls on $L$. japonicum has been considered to be different from $A$. sphaera that is responsible for fruit galls on the same host plant (Yukawa \& Masuda, 1996). In the present paper, however, they are treated as an identical species because our preliminary DNA analysis indicated their similarity (unpublished data).

Fruit gall on Ligustrum obtusifolium Sieb. et Zucc. by Asphondylia sphaera Monzen

[HS: Chiba]: Hamaogi, Amatsukominato Town, 31 Mar. 2002, JY; [KY: Fukuoka]: Kyushu Univ. Forest, Sasaguri Town, 10 Apr. 2001+, NU.

Fruit gall on Osmanthus heterophyllus (G. Don) P. S. Green by Asphondylia sp.

[KY: Fukuoka]: Kyushu Univ. Forest, Sasaguri Town, 10 Apr. 2001+, NU; Maizuru Park, Fukuoka City, 7 May 2001+, NU; [KY: Nagasaki]: Suwa Shrine, Nagasaki City, 25 Mar. 1983, SU. 


\section{RUBIACEAE}

Flower bud gall on Paederia scandens (Lour.) Merril by Asphondylia sp.

[KY: Fukuoka]: Inunaki, Wakamiya Town, 8 Jul. 1999, NU; Kyushu Univ. Forest, Sasaguri Town, 19 Sep. $2000+$, NU.

\section{VERBENACEAE}

Fruit gall on Callicarpa japonica Thunb. (Fig. 1D) by Asphondylia sp.

[KY: Kagoshima]: Shiroyama, Kagoshima City, 7 Jun. 2000, JY; Nishinoomote City, Tanegashima Is., 7 Jun. 2000, K. Ogata.

Most of galls were parasitized by eurytomid wasps. See remarks for further details.

\section{CAPRIFOLIACEAE}

Bud gall on Weigela coraeensis Thunb. by Asphondylia diervillae Felt

[HS: Chiba]: Hamaogi, Amatsukominato Town, 31 Mar. 2002, JY; [KY: Fukuoka]: Aobanomori Park, Fukuoka City, 7 May 2001; Shikanoshima Is., 20 May 2001, NU; Rikimaru Dam, Wakamiya Town, 14 May 2001, NU; [KY: Nagasaki]: Nitta Pass, Unzen (alt. 1100m), 25 Apr. 2001, NU; [KY: Kumamoto]: Mt. Ichifusayama, Mizukami Vil., 4 May 2001, JY.

Bud gall on Weigela decora Nakai [New host rec.] by Asphondylia diervillae Felt

[HS: Akita]: Mt. Ohmoriyama, Higashi-Naruse Vil., 19 Sep. 2001, JY; [HS: Miyagi]:

Nuruyu, Hanayama Vil., 18 Sep. 2001, JY; [KY: Fukuoka]: Minami Park, Fukuoka City, 30 May 2000, NU.

Bud gall on Weigela hortensis K. Koch by Asphondylia diervillae Felt

[HK]: Mt. Moiwayama, Sapporo City, 26 Jun. 2001*, JY; [KY: Fukuoka]: Nagatani Park, Fukuoka City, May 1999+, NU; Minami Park, Fukuoka City, 30 May 2000, NU.

\section{ZINGIBERACEAE}

Fruit gall on Alpinia intermedia Gagnep by Asphondylia sp.

[KY: Kagoshima]: Anjou, Nishinoomote City, Tanegashima Is., 24 Oct. 1997, K.

Ogata; Satamisaki, Sata Town, 11 Apr. 2000*, JY; [SW: Okinawa]: Hijigawa River, Kunigami Vil., 24 Feb. 2001*+, JY; Yona, Kunigami Vil., 24 Mar. 2000*+, NU.

\section{Remarks}

The collection records of Asphondylia gall midges on the following host plants were excluded from the aforementioned data, because they will be referred to in Yukawa et al. (in preparation) that deals with the host alternation by the soybean pod gall midge: fruit gall on Prunus zippeliana Miq. (ROSACEAE); pod gall on Glycine max (L.) Merril, Glycine usuriensis Regel et Maack., and Lespedeza bicolor Turcz. (FABACEAE); flower bud gall and fruit gall on Hedera rhombea (Miq.) Bean (ARALIACEAE). 
Table 1. Distributional range of Asphondylia gall midges in Japan.

\begin{tabular}{|c|c|c|c|c|c|c|c|}
\hline \multirow[b]{2}{*}{ Gall midge } & \multirow[b]{2}{*}{ Host plant } & \multirow[b]{2}{*}{ Hokkaido } & \multicolumn{2}{|c|}{ Honshu } & \multirow[b]{2}{*}{ Shikoku } & \multirow[b]{2}{*}{ Kyushu } & \multirow{2}{*}{$\begin{array}{c}\text { South of } \\
\text { Yakushima Is }\end{array}$} \\
\hline & & & Tohoku & $\begin{array}{l}\text { South of } \\
\text { Tohoku }\end{array}$ & & & \\
\hline \multicolumn{8}{|l|}{ Univoltine gall midges } \\
\hline $\begin{array}{l}\text { Asphondylia } \\
\text { morivorella }\end{array}$ & $\begin{array}{l}\text { Morus spp. } \\
\text { [MORACEAE] }\end{array}$ & - & ? & (e) & - & - & - \\
\hline $\begin{array}{l}\text { Asphondylia } \\
\text { aucuba }\end{array}$ & $\begin{array}{l}\text { Aucuba japonica } \\
\text { [CORNACEAE] }\end{array}$ & - & - & ? & a & - & - \\
\hline $\begin{array}{l}\text { Asphondylia } \\
\text { sphaera }\end{array}$ & $\begin{array}{l}\text { Ligustrum spp. } \\
\text { [OLEACEAE] }\end{array}$ & - & 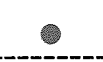 & - & - & - & 도․ \\
\hline \multicolumn{8}{|c|}{ Multivoltine gall midges utilizing spring-summer hosts } \\
\hline Asphondylia sp. & $\begin{array}{l}\text { Rhus spp. } \\
\quad \text { [ANACARDIACEAE] }\end{array}$ & - & - & - & - & 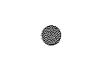 & - \\
\hline Asphondylia baca & $\begin{array}{l}\text { Ampelopsis } \\
\quad \text { brevipedunculata }\end{array}$ & (e) & a & - & 2. & - & (2) \\
\hline & $\begin{array}{l}\text { Cayratia japonica } \\
\text { [VITACEAE] }\end{array}$ & - & - & 웅 & - & - & - \\
\hline Asphondylia sp. & $\begin{array}{l}\text { Helwingia japonica } \\
\text { [CORNACEAE] }\end{array}$ & - & - & 숭 & - & - & - \\
\hline Asphondylia sp. & $\begin{array}{l}\text { Pieris japonica } \\
\text { [ERICACEAE] }\end{array}$ & - & - & - & - & a & - \\
\hline Asphondylia sp. & $\begin{array}{l}\text { Callicarpa japonica } \\
\text { [VERBENACEAE] }\end{array}$ & - & - & - & - & - & - \\
\hline \multicolumn{8}{|c|}{ Multivoltine gall midges utilizing autumn-winter hosts } \\
\hline Asphondylia sp. & $\begin{array}{l}\text { Distylium racemosum } \\
\text { [HAMAMELIDACEAE] }\end{array}$ & - & - & - & - & - & - \\
\hline Asphondylia sp. & $\begin{array}{l}\text { Sapium japonicum } \\
\text { [EUPHORBIACEAE] }\end{array}$ & - & - & - & - & - & - \\
\hline Asphondylia sp. & $\begin{array}{l}\text { Celastrus orbiculatus } \\
\text { [CELASTRACEAE] }\end{array}$ & - & - & - & - & - & - \\
\hline Asphondylia sp. & $\begin{array}{l}\text { Ardisia japonica } \\
\text { [MYRSINACEAE] }\end{array}$ & - & 울 & 중 & - & - & - \\
\hline Asphondylia sp. & $\begin{array}{l}\text { Osmanthus heterophyllus } \\
\text { [OLEACEAE] }\end{array}$ & - & - & (2) & - & - & - \\
\hline Asphondylia sp. & $\begin{array}{l}\text { Paederia scandens } \\
\text { [RUBIACEAE] }\end{array}$ & $\cdot$ & - & - & - & - & - \\
\hline $\begin{array}{l}\text { Asphondylia } \\
\text { diervillae }\end{array}$ & $\begin{array}{l}\text { Weigela spp. } \\
\text { [CAPRIFOLIACEAE] }\end{array}$ & - & - & ? & 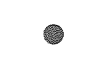 & 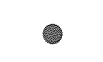 & - \\
\hline Asphondylia sp. & $\begin{array}{l}\text { Alpinia intermedia } \\
\text { [ZINGIBERACEAE] }\end{array}$ & - & - & - & - & - & - \\
\hline
\end{tabular}

Table 1 shows the distribution range of univoltine and multivoltine Asphondylia gall midges in Japan. The univoltine gall midges can complete their annual life history on a known single host plant species or genus, no matter where they are distributed in. However, we need to combine spring-summer gall midges with autumn-winter gall midges to make their annual life cycle complete. Most of the multivoltine gall midges are distributed commonly in Honshu and Kyushu. Only two of them have been recorded from Shikoku, but further surveys will confirm their occurrence, because many other gall midges that are distributed both in Honshu and in Kyushu are found also in Shikoku (Yano, 1964; Yukawa, 1976; Yukawa, 1977).

Therefore, we focused on the presence or absence records in Hokkaido and on the Southwestern Islands south of Yakushima Island. From Table 1, we suspect the combination 
between $A$. baca and $A$. diervillae because they are only multivoltine gall midges distributed in Hokkaido. The galls of $A$. diervillae have never been found on the Southwestern Islands, as species of the genus Weigela (its host plants) are not distributed on the islands (Satake et al., 1989). However, A. baca is considered to complete its annual life cycle only on Ampelopsis brevipedunculata because we observed its fruit galls even in February on these islands.

As mentioned earlier, the fruit gall on Callicarpa japonica caused by an Asphondylia gall midge is different in appearance and inner structure from that recorded in Yukawa \& Masuda (1996). This gall midge was collected from Kyushu (Kagoshima Prefecture) and the Southwestern Islands. A similar distribution pattern was found also for an Asphondylia gall midge on Alpinia intermedia. We must keep this pattern in mind for establishment of further combinations.

As to the remaining gall midges, we could not divide them into species group to determine possible combinations due to the overlapping of their distribution ranges.

\section{Acknowledgements}

We wish to express our thanks to the following persons for their help in collecting galls and giving us information on the Asphondylia gall midges: Dr. S. Akimoto (Hokkaido Univ.), Mr. K. Goukon (Tohoku Gakuin Univ.), Prof. Y. Iokawa (Joetsu Univ. of Education), Mr. S. Usuba (Saitama City), Mr. K. Ogata (Nishinoomote City), Mr. M. Yukinari (previously in Tokushima Agr. Exp. Stn.), Mr. F. Kawamura (Okinawa Pref. Agr. Stn.), Mr. Y. Tohsaka (Kyoto Univ.), Mr. S. Fukuda (Hachinohe City), Ms. E. Kimura (Aomori City), Mr. S. Sato, Miss M. Shoubu, and Mr. H. Inoue (Kyushu Univ.). Nami Uechi and Makoto Tokuda also thank Dr. O. Tadauchi, Dr. S. Kamitani, and Mr. D. Yamaguchi (Kyushu Univ.) for their encouragement.

\section{References}

Satake, Y., H. Hara, S. Watari \& T. Tominari, 1989. Wild Flowers of Japan. Woody Plants II. Heibonsha, Tokyo, 303 pp. (In Japanese.)

Yano, T., 1964. [Insect galls in Matsuyama City and its vicinity]. Ehime Koukou-Rika 1: 66-80.

Yukawa, J., 1971. A revision of the Japanese gall midges (Diptera: Cecidomyiidae). Mem.Fac. Agr. Kagoshima Univ., 8: 1-203.

Yukawa, J., 1976. Check list of midge galls of Japan, with descriptions of newly recorded galls, I. Choripetalae. Mem. Fac. Agr. Kagoshima Univ., 12: 109-123.

Yukawa, J., 1977. Check list of midge galls of Japan, with descriptions of newly recorded 
galls,II. Plants other than Choripetalae. Mem. Fac. Agr. Kagoshima Univ., 13: 8999.

Yukawa, J., 1982. Distribution of the Japanese species of the genus Asphondylia (Diptera, Cecidomyiidae), including the soybean pod gall midge. Proc. Assoc. Pl. 9 Prot. Kyushu, 28: 166-169.

Yukawa, J. \& H. Masuda, 1996. Insect and Mite Galls of Japan in Colors. Zenkoku Nôson Kyôiku Kyôkai, Tokyo, 826 pp. (In Japanese, with English explanations for color plates.) 